\title{
Selective Generation of Charge-Dependent / Independent Ion Energy Distributions From a Heated Capillary Electrospray Source
}

\author{
Victoria L. Campbell, Ziqiang Guan, and David A. Laude, Jr. \\ Department of Chemistry and Biochemistry, University of Texas at Austin, Austin, Texas, USA
}

\begin{abstract}
Retarding grid and Fourier transform ion cyclotron resonance (FIICR) mass spectrometry variable trap potential measurements are performed to determine factors that contribute to the kinetic energy distribution of ions formed in an electrospray source that uses a heated capillary for desolvation. The control of ion kinetic energies is achieved by manipulating the skimmer position in the postcapillary expansion and by varying the potential applied to the skimmer. The selective generation of either charge-dependent or charge-independent ion energy distributions is demonstrated. Charge-dependent energy distributions of electrosprayed ions are created by sampling ions near the Mach disk of the supersonic expansion and by using a larger diameter skimmer orifice; the FIICR spectra acquired under these conditions exhibit mass-to-charge ratio-dependent mass discrimination determined by the potential used to trap the ions. Charge-independent energies of electrosprayed ions are created by positioning the capillary adjacent to the skimmer to sample thermal ions and by using a smaller skimmer orifice to reduce expansion cooling; under these conditions ion kinetic energy is determined primarily by the skimmer potential and no mass-to-charge ratio-dependence is observed in the selection of optimum FTICR trapping conditions. The ability to select between proteins of different conformation on the basis of kinetic energy differences is demonstrated. For example, a $0.4 \mathrm{~V}$ difference in trap potential is observed in the selective trapping of open and closed forms of the +10 charge state of lysozyme. Finally, it is demonstrated that by operating the source under conditions which deliver a beam of ions with charge-independent energies to the cell, it is possible to obtain precursor and product ion signal magnitudes in FTICR spectra without charge-dependent mass discrimination. () Am Soc Mass Spectrom 1994, 5, 221-229)
\end{abstract}

$\mathrm{T}$ There is a recent interest in better understanding and controlling the distribution of ion kinetic energies in electrospray ionization (ESI) mass spectrometry, both as a means to achieve charge- or compound-dependent selectivity in delivering electrosprayed ions to the mass analyzer [1-3], and also as a means to improve sensitivity in ion trap mass analyzers [4-11]. For example, in Fourier transform ion cyclotron resonance (FTICR) mass spectrometry, the elecIrospray ions must be captured in the trapped ion cell prior to detection, a process that requires the potential well depth established between the trapping electrodes to exceed the maximum ion kinetic energy per unit charge of the electrospray ion in the cell [12]. Optimum FTICR performance is achieved when the trapping electric field is small, typically fractions of a volt per centimeter, corresponding to a trap potential of a few volts for cells of standard size and geometry. Thus, for the FTICR detection of electrospray ions to be success-

Address reprint requests to David A. Laude, Department of Chemistry and Biochemistry, University of Texas at Austin, Austin, TX 78712 . ful, the ions must either possess a kinetic energy of a few electron volts per elementary charge upon exiting the source or be decelerated prior to arrival at the cell. These conditions have been achieved and ESI/FTICR is now widely demonstrated [5-11].

The coupling of high pressure external ion sources to FTICR is generally accumplished with an interface that combines multiple stages of differential pumping with ion injection optics designed to guide the ions across the magnetic field gradient and into the cell [13-20]. Because injection efficiency improves with an increase in the forward kinetic energy of the externally formed ions, large external fields often are used to increase ion velocity. However, it then becomes necessary to decelerate ions prior to capture [18]. Alternatively, quadrupolar lens assemblies are used to guide ions through the fringing magnetic field at lower kinetic energies [16]. With either approach to ion injection, the consequence of additional focusing optics is that the ion kinetic energies derived from the electrospray source are convoluted with contributions from subsequent electric fields and a broadened kinetic energy distribution arrives at the cell. For example, the 
kinetic energy distributions for electrospray ions formed in external sources and transmitted to the cell include near-gaussian profiles of $15 \mathrm{eV} / \mathrm{q}$ and a full width at half maximum (FWHM) of $12 \mathrm{eV}$ [10] or 8 $\mathrm{eV} / \mathrm{q}$ and an FWHM of $3 \mathrm{eV}$ [11]. The resulting increase and broadening of the kinetic energy distribution does not preclude the acquisition of high resolution and high sensitivity spectra, but trapping efficiency is reduced and selectivity advantages [3] derived from a more precise control of the kinetic energy suffer.

As an alternative to the external source configurations described above, we have coupled ESI to FTICR with the spray formed in the strong magnetic field within a few centimeters of the cel] $[8,9]$. Two advantages with respect to transfer of ions to the cell are that the magnetic field gradient is not encountered and the radial kinetic energy of ions exiting the spray is confined by the magnetic field. Consequently, elaborate focusing of ions to effect efficient transfer to the cell is unnecessary, and instead ions arrive at the cell with a kinetic energy distribution representative of electrospray source parameters.

In our source design the total ion kinetic energy is derived from two controllable contributions: the flow dynamics in the spray and the electric field generated by applying a potential to the skimmer. These are expressed as the first and second terms, respectively, in eq 1 which predicts the optimum FTICR trapping potential, $V_{\text {trap, }}$ for maximizing the number of ions retained in the cell [3].

$$
\mathrm{V}_{\text {trap }}=\mathrm{C}\left(\mathrm{Av}^{2} / 2 \mathrm{e}\right)(m / z)+\mathrm{K}_{\mathrm{eff}} \mathrm{V}_{\text {skim }}
$$

In the first term, $\mathrm{C}$ is a conversion factor in electron volts per joules, $\mathrm{A}$ is a conversion factor in atomic mass units per kilogram, $v$ is the ion velocity in the expansion, $\mathrm{e}$ is the elementary charge in Coulombs, $m$ is the mass in kilograms, and $z$ is the number of elementary charges. In the second term, $K_{\text {eff }}$ is the correction factor for the effective field experienced by the ions and $V_{\text {skim }}$ is the potential applied to the skimmer. It is important to note that in eq 1 the kinetic energy derived in the first term from the gas expansion is mass-to-charge ratio-dependent in contrast with the kinetic energy derived in the second electric field term which is mass-to-charge ratio-independent. From eq 1 the relative contribution of each energy term is obtained by plotting the optimum trapping potential for each charge state. The expansion contribution to the ion velocity is obtained from the slope, and the effective electric field experienced by the ions is obtained from the y-intercept.

In this article we intend to demonstrate that full control of electrospray ion kinetic energy is achieved by manipulating the ion skimmer orifice diameter, the skimmer position in the postcapillary expansion, and the potential applied to the skimmer. Ion populations with either charge-dependent or charge-independent energies may be selectively generated and delivered to the mass analyzer for detection.

\section{Experimental}

All mass spectra and kinetic energy data were obtained with an FTICR mass spectrometer coupled to an ESI source. Details of the spectrometer configuration and the ESI source are presented elsewhere $[3,8,9]$. Briefly, the spectrometer included a $3.0 \mathrm{~T}$ magnet with four concentric vacuum chambers of increasing diameter extending into the high-field region of the magnet. Electrospray ionization was performed by applying a potential of $3.7 \mathrm{kV}$ to the $100 \mu \mathrm{m}$ i.d. bluntended syringe needle. The syringe needle was positioned 6 $\mathrm{mm}$ from the $500 \mu \mathrm{m}$ i.d. desolvating capillary, $20 \mathrm{~cm}$ in length, which was biased to $330 \mathrm{~V}$ and resistively heated to $\equiv 105^{\circ} \mathrm{C}$ by an applied current of $2.2 \mathrm{~A}$.

Presented in Figure 1 are two separate configurations of the spray and skimmer assembly with indicated pressure readings at various capillary-skimmer distances. In Figure 1a, the electrospray source features a skimmer with a $500 \mu \mathrm{m}$ diameter orifice. The capillary-skimmer region of this arrangement is pumped to 8 torr. In Figure 1b, the electrospray source features a reduced skimmer orifice of $250 \mu \mathrm{m}$ and is

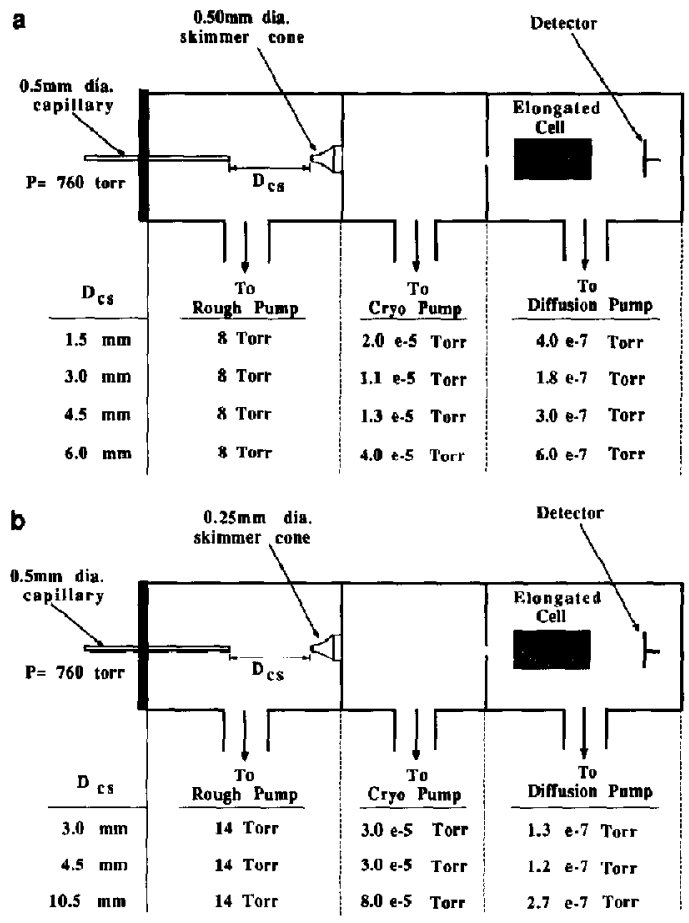

Figure 1. Electrospray source configurations: (a) older design with $500 \mu \mathrm{m}$ diameter skimmer used to promote chargedependent electrospray ion beams and (b) newer design with 250 $\mu \mathrm{m}$ diameter skimmer used to promote charge-independent electrospray ion beams. 
pumped to a pressure of 14 torr in the roughing region. In both cases the postskimmer region was pumped by an $1100 \mathrm{~L} / \mathrm{s}$ cryopump to pressures in the $10^{-5}$ torr range. The last stage of differential pumping was achieved by using both a $1200 \mathrm{~L} / \mathrm{s}$ diffusion pump and a $900 \mathrm{~L} / \mathrm{s}$ diffusion pump, reducing pressures to the low $10^{-7}$ torr range. An elongated trapped ion cell [21-23] with $x, y$, and $z$ dimensions of 5 $\mathrm{cm} \times 5 \mathrm{~cm} \times 10 \mathrm{~cm}$, respectively, and $90 \%$ transmissive mesh trap plates was positioned in the analyzer chamber to collect ions for FTTCR detection. Finally, a solid collector plate was positioned $5 \mathrm{~cm}$ beyond the back trap plate and used in conjunction with it to obtain retarding grid data.

Electrospray parameters. Solutions were fed to the syringe needle through a 22-gauge Teflon tube that was connected to an Isco-500 (Isco Inc., Lincoln, NE) microflow syringe pump delivering a flow rate of 4 $\mu \mathrm{L} / \mathrm{min}$. Solutions were prepared by dissolving the sample in an aqueous solution of acetic acid and then diluting with methanol to achieve a final solution concentration of 5 to $10 \mathrm{pmol} / \mu \mathrm{L}$ in a solution with a volume ratio of 30:68:2 $\mathrm{H}_{2} \mathrm{O}-\mathrm{MeOH}-\mathrm{HOAc}$. Horse heart myoglobin (molecular weight 16,951), cytochrome $c$ (molecular weight 12,360), bee venom melittin (molecular weight 2845), and chicken egg white lysozyme (molecular weight 14,305) were used as obtained from Sigma Chemical Co. (St. Louis, MO).

Data acquisition. Data acquisition and processing were accomplished with a Nicolet (Madison, WI) 1280 computer running Extrel FTMS software version 6.0 (Extrel, Madison, WI). Careful control of skimmer and trap electrode potentials was essential, therefore external power supplies were used. A pulse sequence was written to permit the injection of electrospray ions during a $500 \mathrm{~ms}$ accumulation period. A $1 \mathrm{~s}$ cooling time preceded the swept excitation event and broadband detection.

In our experiment the trap plate potentials were held constant as ions with sufficient kinetic energy to overcome the first potential barrier were trapped in the potential well [12]. Among the mechanisms suggested by which ions could be trapped in the cell are ion-neutral collisions, deshielding in the cell of ions entrained in the ion beam [24], and the redirection of ion trajectories so as not to encounter potential depressions at the trap plates. In earlier electrospray work we demonstrated that collisional deceleration is the primary vehicle for trapping electrospray ions [8], which indicates that only those ions with a kinetic energy upon approaching the cell that is slightly in excess of the trapping potential will be captured. FTICR trapping profiles were used to display the ion signal intensity at different trapping potentials. Previously, Freiser and co-workers [25] and Dunbar and Weddle [26] demonstrated that variable trapping profiles could be used to measure the kinetic energy distribution of ions formed in the cell. We observe close overlap between FTICR trapping and retarding grid profiles in our experiment and conclude that variable trapping also can be used to measure kinetic energy distributions for certain types of external ion injection. These data will be the subject of another article.

For retarding grid measurements, the front trap plate was grounded and a variable potential was applied to the rear trap plate while a Keithley (Cleveland, $\mathrm{OH})$ Model 602 electrometer measured current on the collector positioned behind the cell. The kinetic energy distribution of the electrospray ions was then determined by taking the absolute value of the first derivative of collector current with respect to retarding grid voltage.

\section{Results and Discussion}

A variety of electrospray sources are demonstrated in the literature, each with unique kinetic energy characteristics defined by specific features of the source design $[27,28]$. We generalize these electrospray source designs to be of either a Chait-type or Fenn-type source. In the Chait-type source, desolvation of the multiply charged ion population occurs in a heated capillary, and a large rough pump is typically employed at the capillary terminus to achieve a postcapillary pressure in the low torr region [29]. In contrast, the Fern-type source uses a collision gas for desolvation [30], and a large diffusion pump is used at the spray needle terminus to reduce the postneedle pressure to $10^{-5}$ torr.

An important consideration in the sampling of electrosprayed ions is the length of the free-jet expansion from the expansion orifice to the position of the Mach disk. This length is dependent upon the orifice diameter, the pressure in the capillary region prior to the orifice $\left(P_{0}\right)$, and the pressure after the orifice $\left(P_{1}\right)$. If the orifice diameter or the pressure ratio $\left(\mathrm{P}_{0} / \mathrm{P}_{1}\right)$ increases, the expansion length increases. As molecules proceed from the expansion orifice to the Mach disk, they undergo collisions and become rotationally and vibrationally cooled as a narrow velocity distribution is generated [31]. The magnitude of the molecular cooling depends upon the length of the supersonic expansion and the number of collisions. An examination of the supersonic beam literature suggests the Chait source is similar to the Campargue-type supersonic beam [32] which used a large rough pump positioned after the nozzle to achieve pressures in the torr range. Consequently, the free-jet Mach disk is only a few centimeters beyond the expansion nozzle. In contrast, for the Fenn-type source the Mach disk for the free-jet expansion is on the order of tens of centimeters from the nozzle and greater cooling of the electrosprayed ions in the supersonic expansion should be possible [33].

In constructing our ESI mass spectrometer, we found the Chait-type source to be attractive because the 
desolvating capillary is compatible with our probemounted electrospray interface. The additional gas load of the countercurrent gas is avoided and the reduced size of the nozzle-skimmer assembly matches the restrictive geometric considerations associated with operation in the magnet bore. For this reason we confine our evaluation of electrospray source ion kinetic energy to the Chait-type.

Comparison of kinetic energy distributions. As the results will show, we distinguish two regimes for sampling ions that exit the capillary. In one regime, ion acceleration in the expansion is the major contribution to the ion kinetic energy, creating a charge-dependent energy distribution. Charge-dependent energy distributions of electrosprayed ions are obtained by sam pling ions near the Mach disk of the supersonic expansion and by using a larger diameter skimmer orifice; the FTICR spectra acquired under these conditions exhibit charge-dependent mass discrimination determined by the potential used to trap the ions. In the other regime, ion acceleration in the electric field of the skimmer is the major contribution to the ion kinetic energy, creating a charge-independent energy distribution. Charge-independent energies are generated by positioning the capillary adjacent to the skimmer to sample thermal ions and by using a smaller skimmer orifice to reduce expansion cooling; under these conditions ion kinetic energy is determined primarily by the skimmer potential and no mass-to-charge ratiodependence is observed in the selection of optimum FTICR trapping conditions. The ability to maneuver between these distinct sampling regimes provides another degree of freedom in customizing the electrospray experiment for a particular application.

The comparison kinetic energy profiles in Figure 2, acquired from retarding grid measurements of cytochrome $c$ for different skimmer orifice diameters and skimmer potentials in a Chait-type electrospray source, provide evidence of the differences in sampling. As expected from eq 1 , the kinetic energy varies proportionally with $V_{\text {skim }}$. More interestingly, there is a difference in $\mathrm{K}_{\text {eff }}$ that is dependent upon skimmer orifice diameter. Ions exiting the $500 \mu \mathrm{m}$ diameter skimmer exhibit an average kinetic energy that is approximately $30 \%$ of the skimmer potential, whereas ions exiting the $250 \mu \mathrm{m}$ diameter skimmer exhibit a kinetic energy that is about $60 \%$ of the skimmer potential. Another interesting feature of the profiles in Figure 2 is that as the applied skimmer potential decreases, the kinetic energy distribution narrows.

The relative contributions to ion velocity and kinetic energy from gas expansion dynamirs and effertive electric field are demonstrated with the data in Figure 3. The data sets for three different combinations of skimmer orifice and sampling distance are plotted for the optimum FTICR trap potential for FSI/FTICR spectra of individual charge states of cytochrome $c$. According to eq 1 , the ion velocity contribution from

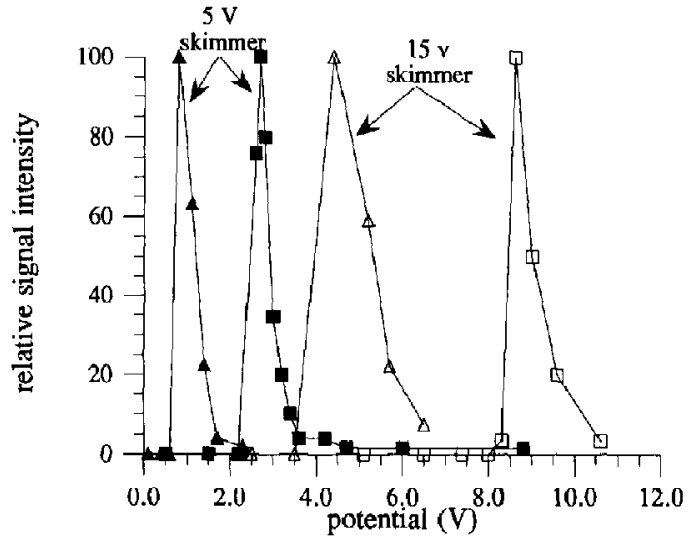

Figure 2. First derivative plots of retarding grid measurements for electrosprayed cytochrome $c$ with a $3 \mathrm{~mm}$ distance between capillary and skimmer: $\Delta$, profile for $15 \mathrm{~V}$ skimmer potential and $500 \mu \mathrm{m}$ diameter skimmer orifice; $\Delta$, profile for $5 \mathrm{~V}$ skimmer and $500 \mu \mathrm{m}$ diameter skimmer orifice; $\square$, profile for $15 \mathrm{~V}$ skimmer potential and $250 \mu \mathrm{m}$ diameter skimmer orifice; profile for $5 \mathrm{~V}$ skimmer and $250 \mu \mathrm{m}$ diameter skimmer orifice. In all measurements the front trap plate was grounded, a variable potential was applied to the second trap plate, and current was measured on a solid plate positioned $5 \mathrm{~cm}$ behind the cell.

the expansion is obtained from the slope and the effective electric field experienced by the ions is obtained from the $y$-intercept. Any kinetic energy contribution from gas dynamics should be distinguished by a nonzero slope, because as the contribution from the expansion increases, the trapping potential will have a greater dependence upon the charge state of the ion. The data in Figure 3 indicate not only that gas dynamics contribute to kinetic energy, but also that the extent

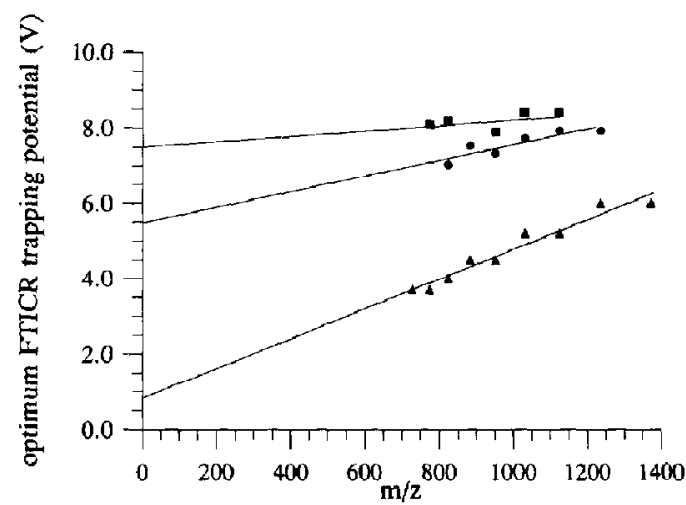

Figure 3. The expansion contribution to ion velocity (Vel) and the effective electric field potential $\left(V_{\text {eff }}\right)$ are calculated from oplimum trapping potentials for individual charge states of cytochrome $c$ with an $11 \mathrm{~V}$ skimmer potential: 4 , using Figure 1a interface with a $500 \mu \mathrm{m}$ skimmer diameter and capillary-skimmer distance $\left(D_{\mathrm{cs}}\right)$ of $10.5 \mathrm{~mm}, \mathrm{Vel}=900 \pm 39 \mathrm{~m} / \mathrm{s}, V_{\text {eff }}=0.8 \pm 1.3$ $\mathrm{V} ;$, using Figure $1 \mathrm{~b}$ interface with a $250 \mu \mathrm{m}$ skimmer diameter and $D_{c s}$ of $9.0 \mathrm{~mm}, \mathrm{Vel}=648 \pm 80 \mathrm{~m} / \mathrm{s}, V_{\text {eff }}=5.5 \pm 0.7 \mathrm{~V}$; using Figure $1 \mathrm{~b}$ interface with a $250 \mu \mathrm{m}$ skimmer diameter and $D_{c s}$ of $4.5 \mathrm{~mm}, \mathrm{Vel}=207 \pm 89 \mathrm{~m} / \mathrm{s}, \mathrm{V}_{\text {eff }}=7.5 \pm 0.7 \mathrm{~V}$. 
of the contribution can be controlled through judicious control of instrumental parameters. For example, by using the larger $500 \mu \mathrm{m}$ skimmer orifice and positioning the skimmer near the Mach disk, both of which promote a supersonic expansion, an estimated velocity of $900 \mathrm{~m} / \mathrm{s}$ is determined for the cytochrome $c$ ions. In contrast, by using a $250 \mu \mathrm{m}$ skimmer diameter and positioning the capillary closer to the skimmer, the velocity achieved by the ions in the expansion could be systematically reduced, with a calculated value of 207 $\mathrm{m} / \mathrm{s}$ shown in Figure 3 .

The data in Figure 3 also demonstrate that protein ions obtain higher velocities as the capillary-skimmer distance is increased. This phenomenon can be attributed to the properties of the free-jet expansion. All molecules that exit the capillary exhibit a thermal kinetic energy that is dependent upon the temperature of the capillary. At the onset of the expansion the solvent molecules, water and methanol, and the large molecular weight proteins all exit the capillary with equivalent energies but different velocities. Over the course of the expansion, the small molecules achieve only a small increase in velocity, but the large protein molecules must undergo a substantial increase in velocity. Figure 3 demonstrates that at a capillaryskimmer distance of $9.0 \mathrm{~mm}$, sampling occurs after ions have traveled a greater distance in the expansion and hence obtain a larger protein velocity of $648 \mathrm{~m} / \mathrm{s}$. In contrast, the earlier sampling at a capillary-skimmer distance of $4.5 \mathrm{~mm}$ does not promote the supersonic expansion and protein velocities drop to $207 \mathrm{~m} / \mathrm{s}$. Interestingly, as the mass-to-charge ratio-dependent expansion contribution to ion velocity decreases, the mass-to-charge ratio-independent effective electric field contribution to ion velocity increases. The data in Figure 3 indicate that it is possible to select experimental conditions that generate either a charge-dependent $\left(\mathrm{v}=900 \mathrm{~m} / \mathrm{s}, \mathrm{V}_{\text {eff }}=0.8 \mathrm{~V}\right)$ or a charge-independent $\left(\mathrm{v}=207 \mathrm{~m} / \mathrm{s}, \mathrm{V}_{\text {eff }}=7.5 \mathrm{~V}\right)$ kinetic energy distribution of electrosprayed ions.

The total kinetic energy of a trapped ion can be determined from eq 2 ,

$$
z \mathrm{eV}_{\text {trap }}-\mathrm{C}\left(\mathrm{mv}^{2} / 2\right)+\mathrm{ze}\left(\mathrm{K}_{\mathrm{eff}} \mathrm{V}_{\text {skim }}\right)
$$

where the first term is the kinetic energy in electron volts obtained in the expansion, and the second term is the kinetic energy in electron volts obtained from the effective electric field of the skimmer. For example, when the $250 \mu \mathrm{m}$ skimmer interface is used in conjunction with a $9.0 \mathrm{~mm}$ capillary-skimmer distance, the +12 charge state of cytochrome $c$ is trapped at a trap potential of approximately $7.7 \mathrm{~V}$. Therefore, the total kinetic energy of the ion is approximately $92.4 \mathrm{eV}$. The kinetic energy of the ion in the expansion is dependent upon the velocity obtained in the expansion. With a velocity of approximately $648 \mathrm{~m} / \mathrm{s}$ in the expansion, the energy obtained in the expansion is $26.9 \mathrm{eV}$. Because $K_{\text {eff }} \times V_{\mathrm{skim}}=V_{\text {eff }}$ and is determined to be ap- proximately $5.5 \mathrm{~V}$, the energy obtained in the effective electric field of the skimmer is about $66.0 \mathrm{eV}$.

Charge-independent ion kinetic energies. A more detailed study of FTICR ion trapping characteristics with the $250 \mu \mathrm{m}$ skimmer orifice was conducted. Presented in Figure 4 are retarding grid profiles of horse myoglobin at different skimmer potentials for a constant capillary-skimmer distance of $4.5 \mathrm{~mm}$. We observe experimentally that $\mathrm{K}_{\text {eff }}$ is independent of skimmer potential at a fixed capillary-skimmer distance. For example, in Figure 4 the electrospray ions exhibit 2.75 $\mathrm{eV}$ of kinetic energy when a $5 \mathrm{~V}$ skimmer potential is applied and, in general, $\mathrm{K}_{\text {eff }}$ is approximately 0.55 for each of the skimmer potentials applied.

Measurements were next performed to determine with more detail the effect of sampling at different capillary-skimmer positions. Earlier work with this interface indicated that adjustments of even fractions of a millimeter would significantly alter FTICR spectral magnitude. This is understandable because an estimation of the Mach disk position for this Campargue-type expansion is on the order of only a few centimeters from the expansion orifice. Thus it should be possible to see substantial differences in kinetic energy distributions in sampling over the first centimeter from the capillary. It would be expected that within the first few millimeters of the capillary protein ions have not yet assumed supersonic velocities and the mass-to-charge ratio-dependent kinetic energy contribution would not be large. In contrast, sampling at an increased displacement from the capillary would allow protein ions to achieve the isovelocity distribution of the solvent and consequently exhibit a significant mass-to-charge ratio-dependence in FTICR spectra acquired at different trap potential values.

Presented in Figure 5 are retarding grid kinetic energy profiles for horse myoglobin acquired with a $250 \mu \mathrm{m}$ skimmer at a potential of $15 \mathrm{~V}$ and capillaryskimmer distances of $3.0 \mathrm{~mm}, 4.5 \mathrm{~mm}$, and $10.5 \mathrm{~mm}$. The dala suggest several significant trends. First, ion kinetic energy increases as capillary-skimmer distance decreases. Second, under these conditions the contribution of the ion expansion velocity to the total ion kinetic energy is smaller than the electric field contribution, which is confirmed by the $9.0 \mathrm{~mm}$ data in Figure 3 where the expansion only contributes $2.0 \mathrm{~V}$ compared to a contribution of $5.5 \mathrm{~V}$ from the skimmer potential. Additionally, as capillary-skimmer distance decreases, $K_{\text {eff }}$ increases. When using a $15 \mathrm{~V}$ skimmer potential, the ions sampled at $3.0 \mathrm{~mm}$ are trapped at a potential of $9.5 \mathrm{~V}$ and experience about $63 \%$ of the applied skimmer potential ( $K_{\text {eff }} \equiv 0.63$ ); the ions sampled at $10.5 \mathrm{~mm}$ are trapped at $5.5 \mathrm{~V}$ and experience only $\sim 37 \%$ of the applied skimmer potential.

These observations can be explained by evaluating the conditions experienced by the ions on either side of the skimmer as they progress along the beam path. Upon exiting the capillary, the ions experience an 

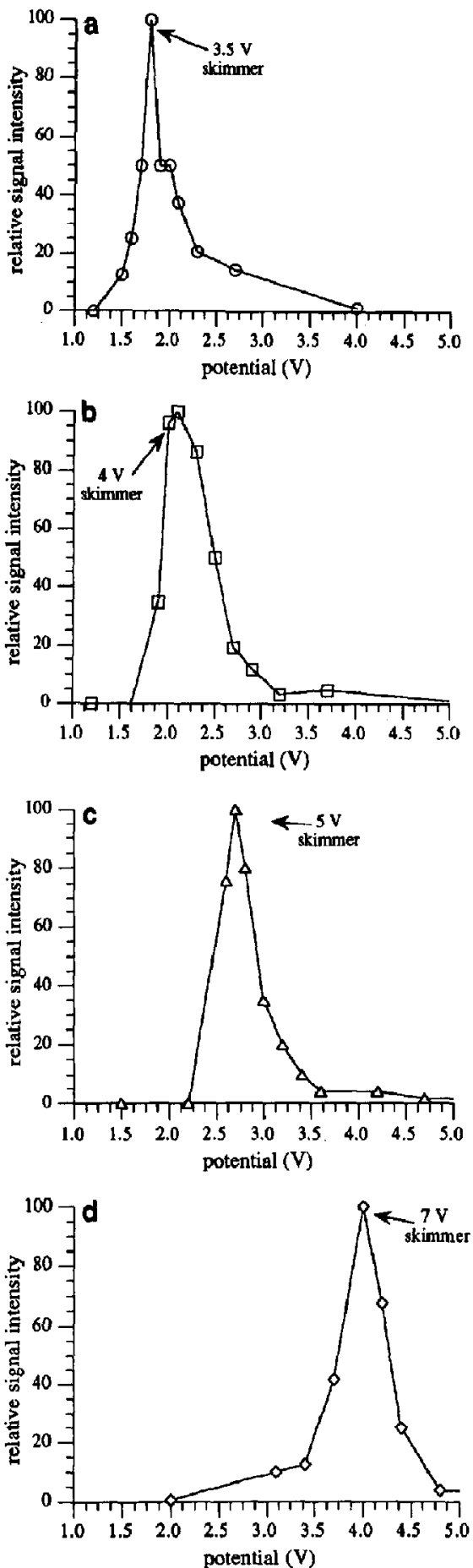

Figure 4. Effect of applied skimmer potential on retarding grid derivative profiles for electrosprayed horse heart myoglobin with a $4.5 \mathrm{~mm}$ distance between capillary and skimmer and $250 \mu \mathrm{m}$ diameter skimmer orifice. The skimmer potential was varied, as shown here, with voltages at (a) $3.5 \mathrm{~V}$, (b) $4 \mathrm{~V}$, (c) $5 \mathrm{~V}$, and (d) $7 \mathrm{~V}$. electric field due to the several hundred volts applied to the capillary. However, this potential was observed to have little effect on the kinetic energy achieved by the ions in this region [9]. Evidently, the large collision rate in proximity to the capillary impedes any ion acceleration in the applied electric field. However, once the ions have penetrated the skimmer orifice, the effective electric field from the skimmer potential appears to be strongly dependent on the capillaryskimmer distance because pressures beyond the skimmer increase with increasing capillary-skimmer distances [9]. One possible reason for the reduction in effective skimmer potential at larger capillary-skimmer displacements is that as the postskimmer pressure increases, the ions do not experience the full accelerating field of the skimmer due to the increased number of collisions and the result is a smaller net kinetic energy from the applied skimmer potential.

Summarizing the data in Figures 3, 4, and 5, if the capillary is positioned adjacent to the skimmer, the expansion is interrupted and ion velocity derived from the expansion is reduced. The substantial pressure in the region between capillary and skimmer prevents any ion acceleration from the applied field derived from the voltage difference between the capillary and skimmer. Therefore only the skimmer potential in the low pressure postskimmer region significantly affects ion kinetic energy. As the capillary-skimmer distance increases, the expansion contribution to the ion velocities increases and ion kinetic energies exhibit a greater mass-to-charge ratio-dependence. In addition, as pressure in the postskimmer region increases, the effective field experienced upon exiting the skimmer region decreases. Thus the net effect of the skimmer potential is reduced at longer capillary-skimmer distances.

Ion conformation effects on ion trapping. There is considerable interest in probing the higher order structure of electrosprayed proteins and other biomolecules by mass spectrometry [34]. One intriguing possibility is that some degree of selection between different conformations of a protein might be feasible. We have already observed that differences in ion kinetic energy can be used to selectively trap individual components of mixtures electrosprayed into the FTICR trapped ion cell [3]. The basis for discrimination of proteins with overlapping mass-to-charge ratio envelopes was that the effective field experienced due to the number of postskimmer collisions and the velocity achieved in the expansion would vary with the shape of the proteins. Thus it is conceivable that, provided sufficient resolution between kinetic energy distributions is achieved, different conformations of the same protein could be selectively trapped at different trap potentials.

Evidence that conformational differences can be distinguished is shown with the variable trapping profiles in Figure 6 for lysozyme in water, in both the closed form and in a more open form after reduction of the four disulfide linkages with 1,4-dithiothreitol. The 


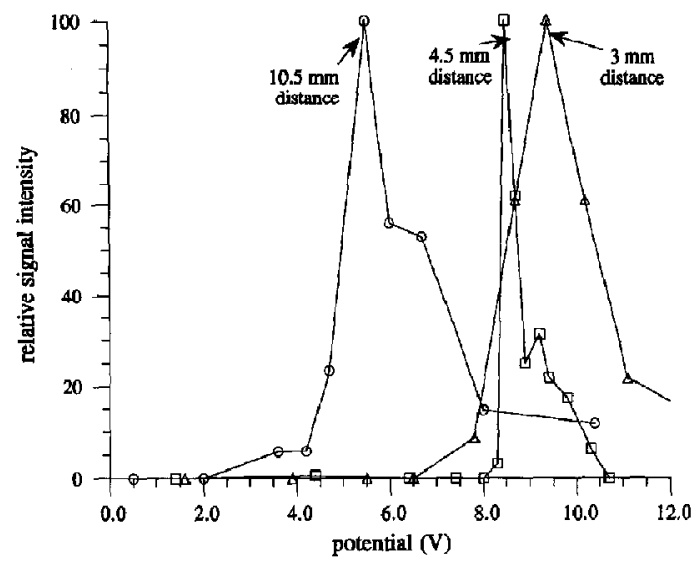

Figure 5. Effect of capillary-skimmer distance on retarding grid derivative profiles for electrosprayed horse heart myoglobin with a $15 \mathrm{~V}$ skimmer potential and $250 \mu \mathrm{m}$ diameter skimmer orifice. The capillary-skimmer distance was varied, as shown here, with distances of $3 \mathrm{~mm}, 4.5 \mathrm{~mm}$, and $10.5 \mathrm{~mm}$.

variable trapping profiles are specifically for the +10 charge state which is abundant in both open and closed forms. Mass-to-charge ratio-dependent effects were excluded by selecting the same charge state and a given nominal mass-to-charge ratio difference of approximately $0.8 \mathrm{Da} / \mathrm{z}$. The profiles indicate that the open form is preferentially trapped at about $0.4 \mathrm{~V}$ lower potential than the closed form. The lysozyme data for the open and closed forms were fit to $\mathrm{eq} I$ and are presented in Figure 7. The data indicate the open, denatured conformation of lysozyme has a greater velocity in the expansion, $359 \mathrm{~m} / \mathrm{s}$, than the closed form which has an expansion velocity of $288 \mathrm{~m} / \mathrm{s}$. In addition, the effective electric field experienced by the open conformer was $0.6 \mathrm{~V}$ less than that of the closed lysozyme ions. Qualitatively, these differences can be explained by the different experiences of the two con-

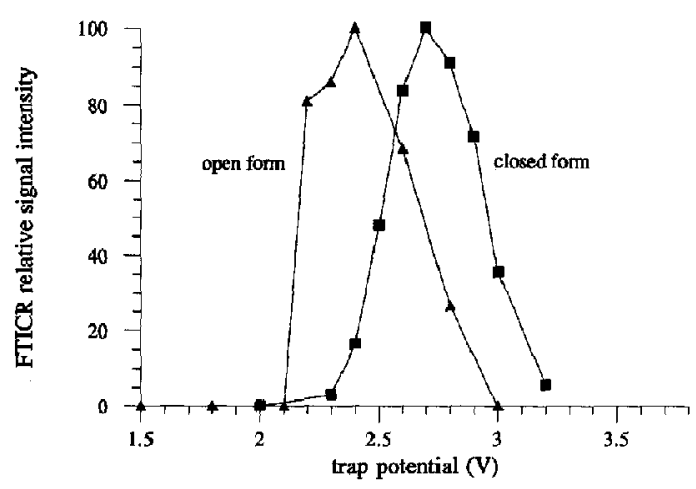

Figure 6. FTICR trapping profiles for open and closed forms of the +10 charge state of lysozyme using Figure $1 \mathrm{~b}$ interface with capillary-skimmer distance of $4.5 \mathrm{~mm}$ and a $4.0 \mathrm{~V}$ skimmer potential. At $2.2 \mathrm{~V}$ and $2.9 \mathrm{~V}$, essentially pure forms of the open $(m / z 1432.3)$ and closed $(m / z$ 1431.5) conformations, respectively, could be selectively trapped in the FIICR cell.

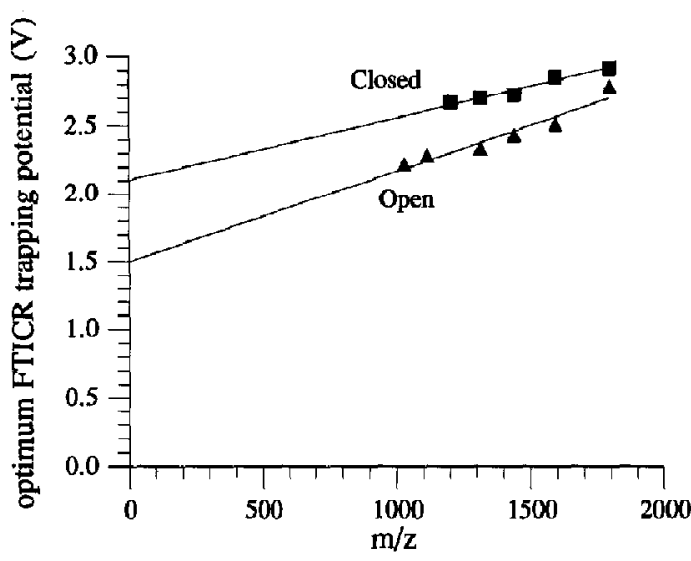

Figure 7. Optimum trapping potentials for individual charge states of the open and closed forms of lysozyme using Figure $1 \mathrm{~b}$ interface with capillary-skimmer distance of $4.5 \mathrm{~mm}$ and a $4.0 \mathrm{~V}$ skimmer potential. The expansion contribution to the ion velocities, Vel, is derived from the slopes of each curve using eq 1 , and ion energy derived from the effective electric field of the skimmer potential, $V_{\text {eff, }}$ is obtained from the $y$-intercept. For the closed conformation, $\mathrm{Vel}=288 \pm 21 \mathrm{~m} / \mathrm{s}$ and $\mathrm{V}_{\text {eff }}=2.1 \pm 0.2 \mathrm{~V}$. For the open conformation, $\mathrm{Vel}=359 \pm 25 \mathrm{~m} / \mathrm{s}$ and $V_{\mathrm{eff}}=1.5 \pm$ $0.3 \mathrm{~V}$. (A $t$-test demonstrates that the two data sets ane different at the $99 \%$ confidence level.)

formations in the supersonic expansion. The open protein conformation should possess an increased collisional cross-section, encounter more unidirectional collisions with solvent molecules in the expansion, and therefore achieve a greater velocity than attained by the smaller, closed conformation. However, the large collisional cross-section that causes an acceleration of the open conformation in the expansion also causes a large number of collisions in the postskimmer region. Because the open-conformation ions will encounter a greater number of collisions than the closed-conformation ions, acceleration in the postskimmer electric field region will be more greatly hindered. Therefore, the open form of lysozyme will have a greater velocity in the expansion, but a smaller $V_{\text {eff }}$. Conversely, the closed form of lysozyme experiences a smaller velocity in the expansion and a larger proportion of the applied skimmer potential, resulting in a larger electric field contribution to its kinetic energy.

Kinetic energy of ESI / FTICR dissociation products. The possibility that multiply charged large biomolecules would be amcnable to ion dissociation is of recent interest [35]. In one technique large desolvating capillary voltages are used to promote fragmentation of exiting electrospray ions to yield dissociation products [36]. However, if a difference in kinetic energy distributions exists for precursor and dissociation product ions, the resulting mass-to-charge ratio discrimination in FTICR spectra would further complicate analysis. Therefore, we used the variable trapping and retarding grid techniques to investigate whether a difference in kinetic energy distributions exists for precursor and 

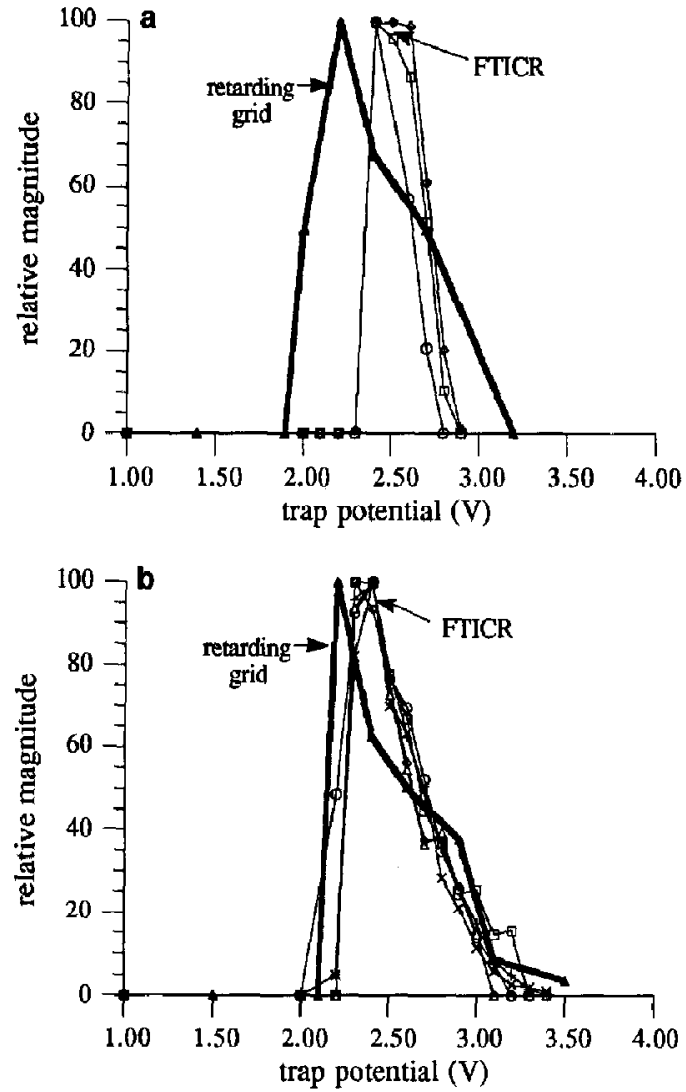

Figure 8. Retarding grid derivative profiles (thick line) and FTICR trapping profiles (thin lines) for electrosprayed melittin with a $5 \mathrm{~V}$ skimmer potential, $4.5 \mathrm{~mm}$ capillary-skimmer distance, and $250 \mu \mathrm{m}$ diameter skimmer orifice. The capillary was heated to promote ion dissociation and FIICR protiles are for all precursor and product ions with the capillary potential at (a) 100 $\mathrm{V}(\mathrm{m} / \mathrm{z} 711,812,948)$ and $(\mathrm{b})$ at $500 \mathrm{~V}(\mathrm{~m} / \mathrm{z} 542,615,671,728$ $812,948)$.

dissociation product ions produced from conditions promoting mass-to-charge ratio-independence. The profiles in Figure 8 are retarding grid and variable trapping profiles of assorted melittin ions generated from $100 \mathrm{~V}$ and $500 \mathrm{~V}$ capillary voltages at a $4.5 \mathrm{~mm}$ capillary-skimmer distance. At a capillary potential of $100 \mathrm{~V}$ the +4 and +3 charge states of melittin, $\mathrm{m} / z$ 711 and 948 , respectively, are generated as well as the $m / z 812$ fragmentation product. As expected, increased fragmentation was observed when $500 \mathrm{~V}$ potential was applied to the capillary. However, the variable trapping profiles for both precursor and product ions at $100 \mathrm{~V}$ and $500 \mathrm{~V}$ were identical, which indicates that even with differences in mass and charge state, no mass-to-charge ratio-dependent trapping occurred. This is likely because sampling at the beginning of the expansion reduces mass-to-charge ratio-dependent contributions to the ion kinetic energy and the optimum trapping conditions for the ions.
The ability to reduce mass-to-charge ratio discrimination in FTICR spectra should simplify analysis of large biomolecules and their dissociation products.

\section{Acknowledgments}

This work is supported by the Welch Foundation (F-1138), the National Institutes of Health, the Texas Advanced Research Program, and the National Science Foundation (CHE9013384 and CHE9057097). We thank Carl Ijames and Steven Hofstadler for helpful discussions.

\section{References}

1. Feng, R.; Konishi, Y. Anul. Chem. 1993, 65, 645-649.

2. Feng, R.; Konishi, Y. Presented at the 12th International Mass Spectrometry Conference; Amsterdam, The Netherlands, August 26-30, 1991

3. Hofstadler, S. A.; Beu, S. C.; Laude, D. A., Jr. Anal. Chem. 1993, 65, 312-316.

4. McLuckey, S. A.; Van Berkel, G. J.; Glish, G. L. J, Am, Chem. Soc. 1990, 112, 5668-5670.

5. Henry, K. D.; McLafferty, F. W. Org. Mass Spectram. 1990, 25, 490-492.

6. Henry, K. D.; Williams, E. R.; Wang, B. H.; McLafferty, F. W.; Shabanowitz, J; Hunt, D. W. Proc. Natl. Acad. Sci. LSA 1989, $86,9075-9078$.

7. Henry, K. D.; Quinn, J. P.; McLafferty, F. W. J. Am. Chem. Soc. 1991, 113, 5447-5449.

8. Hofstadler, S. A.; Laude, D. A., Jr. J. Am. Soc. Mass Spectrom. 1992, 3, 615-623

9. Hufstadler, S. A.; Sclunidt, E.; Guan, Z.; Laude, D. A., Jr. J. Am. Soc. Mass Spectrom. 1993, 4, 168-176.

10. Beu, S. C.; Senko, M. W.; Quinn, J. P.; Wampler, F. M; McLafferty, F. W. I. Am. Soc. Mass Spectrom. 1993, 4, 557-565.

11. Winger, B. E.; Hofstadler, S. A.; Bruce, J. E.; Udseth, H. R.; Smith, R. D. I. Am. Soc. Mass Spectrom. 1993, 4, 566-577.

12. Hofstadler, S. A.; Laude, D. A., Jr. Int. J. Mass Spec. Ion Proc. $1990,101,65-78$.

13. Kofel, P.; Alleman, M.; Kellerhals, Hp.; Wanczek, K. P. Int. J. Mass Spec. Ion Proc. 1989, 87, 237-247.

14. Kofel, P.; Alleman, M.; Kellerhals, Hp.; Wanczek, K. P. Int. J. Mass Spec. Ion Proc. 1985, 65, 97-103.

15. Kofel, P,; Alleman, M.; Kellerhals, Hp.; Wanczek, K. P. Int. J Mass Spec. Ion Proc. 1986, 72, 53-61.

16. Lebrilla, C. B.; Amster, I. J.; McJver, R. T., Jr. Int. J. Mass Spec. Lon Proc, 1989, 87, R7-R13.

17. Alford, J. M.; Williams, P. E; Trevor, D. J.; Smalley, R. E. Int J. Mass Spec. Ion Proc. 1986, 72, 33-51.

18. Smalley, R. E. And. Instrun. 1988, 17, 1-21.

19. Maruyama, S.; Anderson, L. R.; Smalley, R. E. Rev. Sci. Instrum, 1990, 61, 3686-3693.

20. Alford, J. M.; Weiss, F. D; Laaksonen, R. T.; Smalley, R. E. I. Phys. Chem. 1986, 90, 4480-4482.

21. Hunter, R. L.; Sherman, M. G.; Mclver, R. T., Jr. Int. J. Mass Spec, and lon Phys. 1983, 50, 259-274.

22. Hofstadler, S. A.; Laude, D. A., Jr. J. Am. Soc. Mass Spectrom. $1990,1,351-360$.

23. Van De Guchte, W. J.; Van Der Hart, W. J. Int. J. Mass Spec. Ion Proc. 1992, 113, 59-79.

24. Beu, S. C.; Hendrickson, C. L; Vartanian, V. H,; Laude, D. A, Jr. Int. I. Mass Spec. Ion Proc. 1992, 113, 59-79.

25. Gord, J. R.; Freiser, B. S.; Buckner, S. W. I. Chem. Phys. 1991, $94,4282-4290$.

26. Dunbar, R. C.; Weddle, G. H. J. Phys. Chem. 1988, 92, 5706-5709. 
27. Fenn, J. B.; Mann, M.; Meng, C. K.; Wong, S. F.; Whitehouse, C. M. Mass Spectrom. Rev. 1990, 9, 37-70.

28. Smith, R. D.; Loo, J. A.; Edmonds, C. C.; Barinaga, C. J.; Udseth, H. R. Anal. Chem. 1990, 62, 882-899.

29. Chowdhury, S. K.; Katta, V.; Chait, B. T. Rapid Commun. Mass Spectrom. 1990, 1, 91-97.

30. Yamashita, M.; Fenn, J. B. J. Phys. Chem. 1984, 88, 4451-4459.

31. Hayes, J. M.; Small, G. J. Anal. Chem. 1983, 55, 565A-574A.

32. Campargue, R. J. Phys. Chem. 1984, 88, 4466-4474.
33. Levy, D. H.; Wharton, L.; Smalley, R. E. In Chemical and Biochemical Applications of Lasers, vol 2; Academic Press: New York, 1977; chapter 1.

34. Suckau, D.; Shi, Y.; Beu, S. C.; Senko, M. W.; Quinn, J. P.; Wampler, F. M.; McLafferty, F. W. Proc. Natl. Acad. Sci. USA 1993, 90, 790-793.

35. Barinaga, C. J.; Edmonds, C. G.; Udseth, H. R.; Smith, R. D. Rapid Commun. Mass Spectrom. 1989, 3, 160-164.

36. Smith, R. D.; Loo, J. A.; Barinaga, C. J.; Edmonds, C. G.; Udseth, H. R. J. Am. Soc. Mass Spectrom. 1990, 1, 53-65. 